\title{
Left Ventricle Quantification in 3D Echocardiography Using A Geometric Deformable Model
}

\author{
X Hang ${ }^{1}$, NL Greenberg ${ }^{2}$, JD Thomas ${ }^{2}$ \\ ${ }^{1}$ California State University-Northridge, USA \\ ${ }^{2}$ Cleveland Clinic Foundation, Cleveland, USA
}

\begin{abstract}
Real-time 3D echocardiography can provide accurate left ventricle volume measurement without geometric assumptions with the assist of a robust segmentation algorithm. A new 3D geometric deformable model is introduced for the purpose by combining 3D Gradient vector flow $(G V F)$ and $3 D$ geodesic active contour model. The new model is successfully applied to left ventricle quantification in $3 D$ echocardiography.
\end{abstract}

\section{Introduction}

Left ventricle volume quantification plays an important role in cardiac function evaluation. Real-time 3D echocardiography, such as Live3D echo used in this study, can provide accurate end-systolic volume (ESV) and end-diastolic volume (EDV) volume measurement without geometric assumptions. A robust 3D segmentation algorithm can greatly assist the task.

Geodesic active contour model [1] is a well-known geometric deformable model, which allows stable boundary detection in images with good contrast. However, the model suffers from boundary gap problem, which is very common in ultrasonic images. In order to address this problem, we proposed a new geometric deformable model [2] by combining the ideas of geometric deformable model and gradient vector flow (GVF) [3]. In this study the new model is extended to 3D and applied for left ventricle quantification in 3D echocardiography.

\section{Method}

GVF, originally introduced as a kind of external fore for parametric deformable model, can be obtained by means of the diffusion of the gradient vector of the edge map. Let

$$
\mathbf{v}(x, y, z)=[u(x, y, z), v(x, y, z), w(x, y, z)]
$$

be $3 \mathrm{D} \mathrm{GVF}$, and it can be obtained by minimizing the following energy functional

$$
E=\iiint \mu|\nabla \mathbf{v}|^{2}+|\nabla f|^{2}|\mathbf{v}-\nabla f|^{2} d x d y d z
$$

where $\mu$ is a parameter which should be set higher with more noise, and $f$ is the edge map, usually defined as

$$
f=\left|\nabla\left(G_{\sigma}(x, y, z) * I(x, y, z)\right)\right|
$$

Via calculus of variation, GVF can be obtained by solving the following Euler equations

$\mu \nabla^{2} u-\left(u-\frac{\partial f}{\partial x}\right)\left[\left(\frac{\partial f}{\partial x}\right)^{2}+\left(\frac{\partial f}{\partial y}\right)^{2}+\left(\frac{\partial f}{\partial z}\right)^{2}\right]=0$

$$
\mu \nabla^{2} v-\left(v-\frac{\partial f}{\partial y}\right)\left[\left(\frac{\partial f}{\partial x}\right)^{2}+\left(\frac{\partial f}{\partial y}\right)^{2}+\left(\frac{\partial f}{\partial z}\right)^{2}\right]=0
$$

$$
\mu \nabla^{2} w-\left(w-\frac{\partial f}{\partial w}\right)\left[\left(\frac{\partial f}{\partial x}\right)^{2}+\left(\frac{\partial f}{\partial y}\right)^{2}+\left(\frac{\partial f}{\partial z}\right)^{2}\right]=0
$$

The diffusion procedure can seal the boundary gaps. More details on GVF can be found in [3].

$$
\begin{aligned}
& \text { Geodesic active contour is extended to 3D [4] as } \\
& \frac{\partial U}{\partial t}=g(c+H)|\nabla U|+\langle\nabla U, \quad \nabla g\rangle
\end{aligned}
$$

where $U$ is a 4D scalar function, whose zero level set is the evolving surface, $g$ mainly functions as the stop force when the evolving surface arrives to the object boundary and defined as

$$
g=\frac{1}{1+f}
$$

$c$ is a constant to increase the speed of convergence, and $M$ is the curvature given by

$$
\begin{aligned}
M= & {\left[\left(\frac{\partial U}{\partial x}\right)^{2} \frac{\partial^{2} U}{\partial y^{2}}+\left(\frac{\partial U}{\partial y}\right)^{2} \frac{\partial^{2} U}{\partial x^{2}}+\left(\frac{\partial U}{\partial x}\right)^{2} \frac{\partial^{2} U}{\partial z^{2}}+\right.} \\
& \left(\frac{\partial U}{\partial z}\right)^{2} \frac{\partial^{2} U}{\partial x^{2}}+\left(\frac{\partial U}{\partial y}\right)^{2} \frac{\partial^{2} U}{\partial z^{2}}+\left(\frac{\partial U}{\partial z}\right)^{2} \frac{\partial^{2} U}{\partial y^{2}}- \\
& \left.2 \frac{\partial U}{\partial x} \frac{\partial U}{\partial y} \frac{\partial^{2} U}{\partial x \partial y}-2 \frac{\partial U}{\partial x} \frac{\partial U}{\partial z} \frac{\partial^{2} U}{\partial x \partial z}-2 \frac{\partial U}{\partial y} \frac{\partial U}{\partial z} \frac{\partial^{2} U}{\partial y \partial z}\right] /\left.\nabla U\right|^{3} .
\end{aligned}
$$

From Eq. (6) we can get $\nabla g=-\nabla f$. Since 3D gradient $\mathbf{v}$ is obtained by diffusion of $\nabla f$, the first step to build the 
new model is to replace $\nabla g$ with $-\mathbf{v}$ in Eq. (5).

The second step is to define a new $\mathrm{g}$ function $g_{\text {new }}$ that should be defined to obtain the magnitude of the gradient of the 3D vector field GVF. Let $\mathbf{J}$ be Jacobin matrix of $\mathbf{v}$ given by

$$
\mathbf{J}=\left[\begin{array}{lll}
\frac{\partial u}{\partial x} & \frac{\partial v}{\partial x} & \frac{\partial w}{\partial x} \\
\frac{\partial u}{\partial y} & \frac{\partial v}{\partial y} & \frac{\partial w}{\partial y} \\
\frac{\partial u}{\partial z} & \frac{\partial v}{\partial z} & \frac{\partial w}{\partial z}
\end{array}\right]
$$

and define $\mathbf{D}$ as

$$
\mathbf{D}=\mathbf{J}^{T} \mathbf{J} \text {. }
$$

Denote the three eigenvalues as $\lambda_{1} \geq \lambda_{2} \geq \lambda_{3}$. It can be shown that the strength of the magnitude of the gradient of GVF can be defined as $\lambda_{1}-\lambda_{3}$ [5]. The function $g_{\text {new }}$ can be defined as

$$
g_{\text {new }}=e^{\lambda_{3}-\lambda_{1}} \text {. }
$$

At last, since mean curvature $M$ can cause singularities [6] during surface evolution, a combined term, $\operatorname{sign}(M)(G+|G|)^{\frac{1}{4}}[6]$ is used to replace $M$, where $G$ is Gaussian curvature given by

$$
\begin{aligned}
G= & \left\{\left(\frac{\partial U}{\partial x}\right)^{2}\left(\frac{\partial^{2} U}{\partial y^{2}} \frac{\partial^{2} U}{\partial z^{2}}-\frac{\partial^{2} U}{\partial y \partial z}\right)+\right. \\
& \left(\frac{\partial U}{\partial y}\right)^{2}\left(\frac{\partial^{2} U}{\partial x^{2}} \frac{\partial^{2} U}{\partial z^{2}}-\frac{\partial^{2} U}{\partial x \partial z}\right)+ \\
& \left(\frac{\partial U}{\partial z}\right)^{2}\left(\frac{\partial^{2} U}{\partial x^{2}} \frac{\partial^{2} U}{\partial y^{2}}-\frac{\partial^{2} U}{\partial x \partial y}\right)+ \\
& 2\left[\frac{\partial U}{\partial x} \frac{\partial U}{\partial y}\left(\frac{\partial^{2} U}{\partial x \partial z} \frac{\partial^{2} U}{\partial y \partial z}-\frac{\partial^{2} U}{\partial x \partial y} \frac{\partial^{2} U}{\partial z^{2}}\right)+\right. \\
& \frac{\partial U}{\partial y} \frac{\partial U}{\partial z}\left(\frac{\partial^{2} U}{\partial x \partial y} \frac{\partial^{2} U}{\partial x \partial z}-\frac{\partial^{2} U}{\partial y \partial z} \frac{\partial^{2} U}{\partial x^{2}}\right)+ \\
& \left.\left.\frac{\partial U}{\partial x} \frac{\partial U}{\partial z}\left(\frac{\partial^{2} U}{\partial x \partial y} \frac{\partial^{2} U}{\partial y \partial z}-\frac{\partial^{2} U}{\partial x \partial z} \frac{\partial^{2} U}{\partial y^{2}}\right)\right]\right\} /\left.\nabla U\right|^{2}
\end{aligned}
$$

As a result the new 3D geometric deformable model is defined as

$$
\begin{aligned}
& \frac{\partial U}{\partial t}=g_{\text {new }}\left[c+\operatorname{sign}(M)|G+| G||^{1 / 4}\right]|\nabla U| \\
& +\langle\nabla U, \quad-\mathbf{v}\rangle \text {. }
\end{aligned}
$$

And level set method [7] is used to numerically solve the model.

\section{Results}

Figure 1 shows the evolving process of a dynamic surface inside the left ventricle. Figure 2 shows how left ventricle is successfully detected by showing the segmented slice images.

In order to validate the new model, we measured 16 volumes ( $8 \mathrm{ESVs}$ and $8 \mathrm{EDVs}$ ) using the new model. And the results were compared with the average of those obtained by physicians using manual tracing method. The regression analysis in Figure 3 shows a good correlation between them $(p<0.001)$.

\section{Conclusion}

A new 3D geometric deformable model is proposed via combining geodesic active contour model and gradient vector flow. Numerical method is realized by level set methods. The model is applied to left ventricle volume measurement in 3D echocardiography and shows accurate segmentation result. The future research may lies in the further validation of the model for left ventricle quantification and the improvement of computational efficiency.

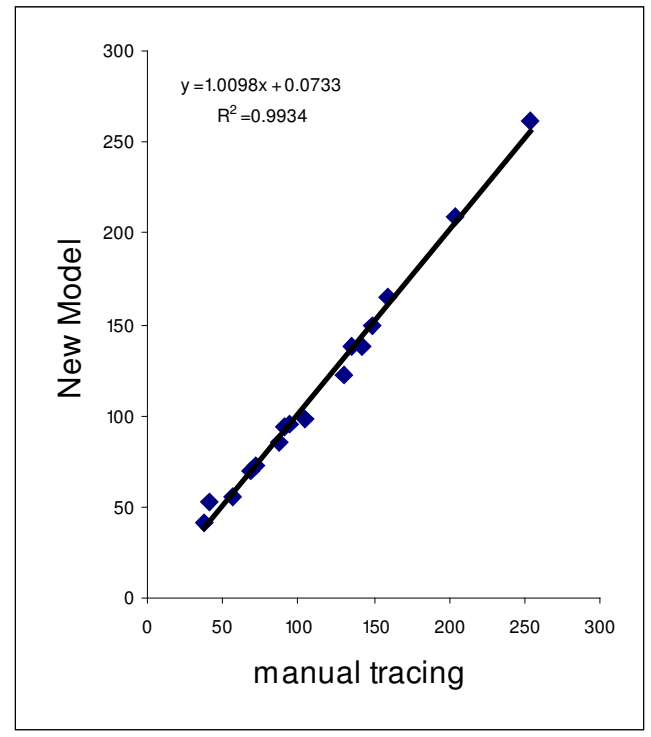

Figure 3: Regression analysis between the volumes obtained by the new model and those by manual tracing. 


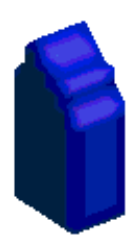

(a) Initialization

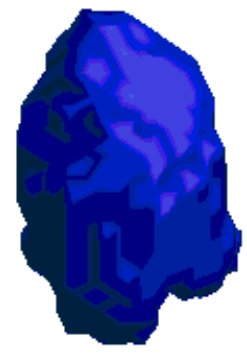

(c) After 30 iterations

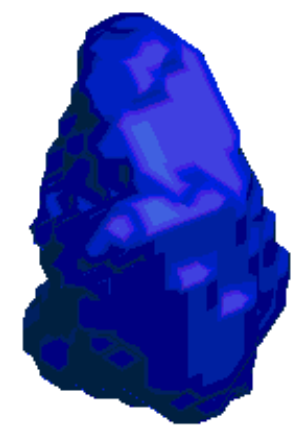

(e) After 70 iterations

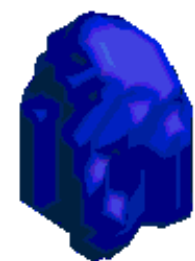

(b) After 10 iterations

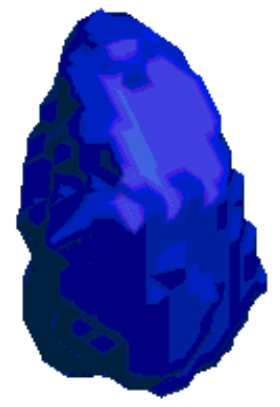

(d) After 50 iterations

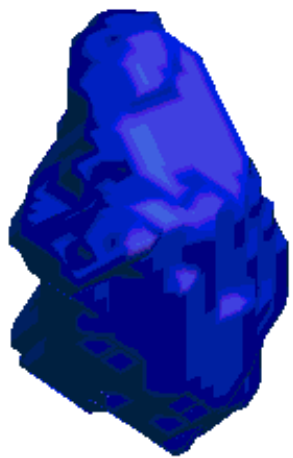

(f) After 180 iterations

Figure 1: Evolving process of a dynamic surface inside the left ventricle 


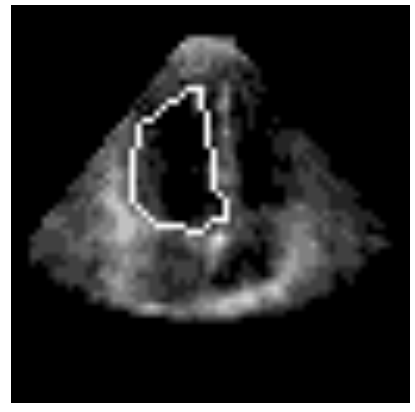

(a)

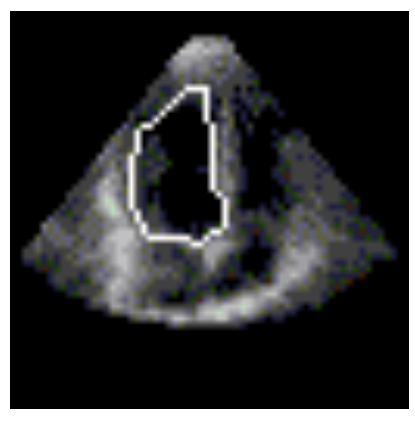

(b)

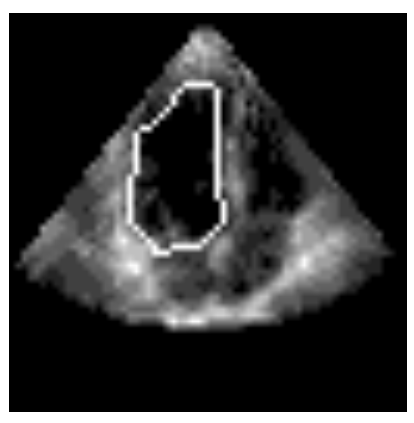

(c)

Figure 2: Segmented slice images

\section{Acknowledgment}

This study was supported by the National Aeronautics and Space Administration (grants NCC9-58 and 60)

\section{References}

[1] Caselles V, Kimmel R, Sapiro G. Geodesic Active Contours. Int. J. Comput. Vis; 1997;22: 61-79.

[2] Hang X, Greenberg NL, Thomas JD. A geometrical deformable model for echocardiographic image segmentation. EEE Computer Society, Los Alamitos, CA; Computers in Cardiology;2002:77-80.

[3] $\mathrm{Xu} \mathrm{C}$, Prince JL. Snakes, shapes, and gradient vector flow. IEEE Trans. Imag. Proc.;1998;7:359-369.

[4] Caselles V, Kimmel R, Sapiro G, and Sbert C. Minimal surfaces based object segmentation. IEEE Trans. Pattern Analysis and Machine Intelligence; 1997;19(4):394-398.

[5] Lee H, Cok DR. Detecting Boundaries in a Vector Field. IEEE Trans. Sig. Proc.; 1991;39:1181-1194.

[6] Caselles V, Sbert C. What is the best causal scale space for three-dimensional images? SIAM J. Applied Math; 1996; 56(4):1199-1246.

[7] Sethian JA. Level Set Methods and Fast Marching Methods, 2nd. Ed. Cambridge, UK: Cambridge University Press; 1999.

Address for correspondence:

Dr. Xiyi Hang

Department of Electrical and Computer Engineering

California State University

18111 Nordhoff Street

Northridge, CA 91325

E-mail address: xhang@csun.edu 